\title{
The Importance of Species Identification in Acinetobacter
}

\section{Hilmar Wisplinghoff ${ }^{1 *}$}

${ }^{1}$ Institute for Medical Microbiology Immunology and Hygiene, University of Cologne, Germany

Acinetobacter species have emerged as one of the most important pathogens in recent years. The clinical relevance of the genus-more precisely several species of this genus-has constantly risen in the past thirty years. Currently the species of the Acinetobacter baumannii group, i.e. A. baumannii, A. pittii (formerly Acinetobacter genomic species 3 ) and $A$. nosocomialis (formerly Acinetobacter genomic species 13), belong to the most resistant organisms known to cause relevant infections in man.

The genus Acinetobacter consists of 31 species, 22 of which have been assigned species names. These species differ widely in their epidemiology, natural habitat, and clinical relevance. While some species such as $A$. schindleri or $A$. ursingii have so far been only isolated from human specimens, others like A. baylyi or A. towneri have only been found in activated sludge or environmental specimens and have never been described as a causative pathogen of an infection in humans.

Much like the genus of the staphylococci where Staphylococcus aureus stands out as one of the clinically most important pathogens, and S. lugdunensis and S. saprophyticus are regularly associated with infections, while most other species of the genus are of minorclinical importance, there are important differences in the clinical relevance of the individual Acinetobacter species.

A. baumannii and to some extend the other members of the A. baumannii group (A. pittii and A. nosocomialis) are important nosocomial pathogens that have been associated with a variety of nosocomial infections, mainly in immunocompromised patients in the ICU setting. Clinical presentation of these infections ranges from benign transient infections to fulminant septic shock and can be associated with high mortality. In contrast, the other Acinetobacter species ("A. non-baumannii species") are of minor clinical relevance and can be regarded a largely non-pathogenic environmental organism.
Unfortunately, in Acinetobacter species, identification to species level is complicated, and none of the routinely used biochemical methods (including the automated systems) is able to clearly differentiate between the individual Acinetobacter species. Reference methods such as specially adopted biochemical panels and molecular methods are either not available or not regularly employed in most of the routine diagnostic laboratories. MALDI-TOF MS may be a solution to this problem, however, on one hand current data are sparse and the results of different studies vary, on the other hand, MALDI-TOF is not yet widely used.

This problem is not only of clinical relevance-here surprisingly less so, since most currently used systems mange to correctly differentiate between A. baumannii group and the other species - but continues to lead to some confusion in the scientific environment.

Several papers-regardless of the quality or impact of the publishing journal-still confuse the pathogenic species A. baumannii (that is almost exclusively isolated from human specimens) with the ubiquitous A. non-baumannii species. Depending on the focus of the studies, incorrect species identification can have a tremendous impact on the results especially if clinical features or resistance rates are reported. Also, most of the epidemiological methods will provide results independently of the investigated species, and problems in the species identification - and therefore the selection of strains to be included in the study - can also considerably alter the results.

In order to expand the knowledge of clinical features and epidemiology of the different Acinetobacter species, all studies should employ reference methods to correctly (re-)identify all included Acinetobacter strains at species level.
*Corresponding author: Hilmar Wisplinghoff, Institute for Medical Microbiology Immunology and Hygiene, University of Cologne, Germany, E-mail: H.Wisplinghoff@wisplinghoff.de

Received August 27, 2012; Accepted August 28, 2012; Published August 30 2012

Citation: Wisplinghoff H (2012) The Importance of Species Identification in Acinetobacter. J Med Microb Diagn 1:e113. doi:10.4172/2161-0703.1000e113

Copyright: (c) 2012 Wisplinghoff $\mathrm{H}$. This is an open-access article distributed under the terms of the Creative Commons Attribution License, which permits unrestricted use, distribution, and reproduction in any medium, provided the original author and source are credited. 